\title{
Screening of differentially expressed genes associated with non-union skeletal fractures and analysis with a DNA microarray
}

\author{
JIAMING XU, CHANGQING ZHANG and WENQI SONG \\ Department of Orthopedics, Shanghai Sixth People's Hospital Affiliated to Shanghai Jiao Tong University, \\ Xuhui, Shanghai 200233, P.R. China
}

Received September 2, 2013; Accepted December 2, 2013

DOI: $10.3892 /$ etm.2014.1478

\begin{abstract}
The purpose of this study was to identify the feature genes that are associated with non-union skeletal fractures using samples of normal union and non-union skeletal fracture microarray data. The gene expression profile GSE494 was downloaded from the Gene Expression Omnibus database and included 12 samples based on three different platforms (GPL92, GPL93 and GPL8300). Each of the platforms had four sets of expression data, two from normal union skeletal fracture samples and two from non-union skeletal fracture samples. The differentially expressed genes within the three platforms of expression data were identified using packages in $\mathrm{R}$ language and the differentially expressed genes common to the three platforms were selected. The selected common differentially expressed genes were further analyzed using bioinformatic methods. The software HitPredict was used to search interactions of the common differentially expressed genes and then FuncAssociate was used to conduct a functional analysis of the genes in the interaction network. Further, the associated pathways were identified using the software WebGestalt. Under the three different platforms, GPL92, GPL93 and GPL8300, the numbers of differentially expressed genes identified were 531, 418 and 914, respectively. The common gene CLU and its interacting genes were most significantly associated with the regulation of sterol transport and the osteoclast differentiation pathway. Upregulation of the gene CLU was identified by comparing data for normal union and
\end{abstract}

Correspondence to: Dr Changqing Zhang or Dr Wenqi Song, Department of Orthopedics, Shanghai Sixth People's Hospital Affiliated to Shanghai Jiao Tong University, 600 Yishan Road, Xuhui, Shanghai 200233, P.R. China

E-mail: changqingzhangcqz@hotmail.com

E-mail: wenqisong@hotmail.com

Key words: non-union skeletal fractures, differentially expressed gene, interaction network, function enrichment analysis, pathway analysis non-union skeletal fracture samples. According to the function of CLU and its interacting genes, it was concluded that they inhibit the normal healing process following a fracture, and result in non-union skeletal fractures through the regulation of sterol transport and the pathways of differentiation in osteoclasts.

\section{Introduction}

There are $>15$ million fractures treated in the United States annually and many more worldwide (1). While the vast majority of these fractures heal with appropriate orthopedic management, $10-15 \%$ of patients suffer complications that result in delayed- or non-union (2). Fracture healing is a multistage repair process that involves complex yet well-established steps that are initiated in response to injury, resulting in the repair and restoration of function (3). Numerous factors have been associated with failure of normal fracture healing, including the fracture location, the extent of soft tissue damage and interposition, the degree of bone loss in anatomic criteria, infection, inadequate reduction, poor stabilization/fixation factors that are exacerbated by treatment, patient characteristics, comorbidities and drug use (2).

Fracture repair involves the pathway of normal embryonic development, which consists of several cell types originating from the cortex, periosteum, surrounding soft tissue and bone marrow space $(4,5)$. Different biological factors, which include recruitment, proliferation and differentiation of cell types, vascular regeneration, expression of growth factors (e.g. IGF, TGF- $\beta$ and BMP) and appropriate biomechanical conditions, have been considered to be critical for the healing of bone fractures. Local imbalances of these different factors during conservative or operative fracture treatment may lead to delay of fracture healing or to fracture non-union (6). According to radiological and histological criteria, non-unions are generally classified into three types (7). Hypertrophic non-unions are often linked with insufficient fracture stability and appear to have an adequate blood, oxygen and nutrient supply, while atrophic non-unions are generally poorly vascularized (7). In defect non-unions, the fracture healing is affected by a lack of contact among fracture fragments (6). 

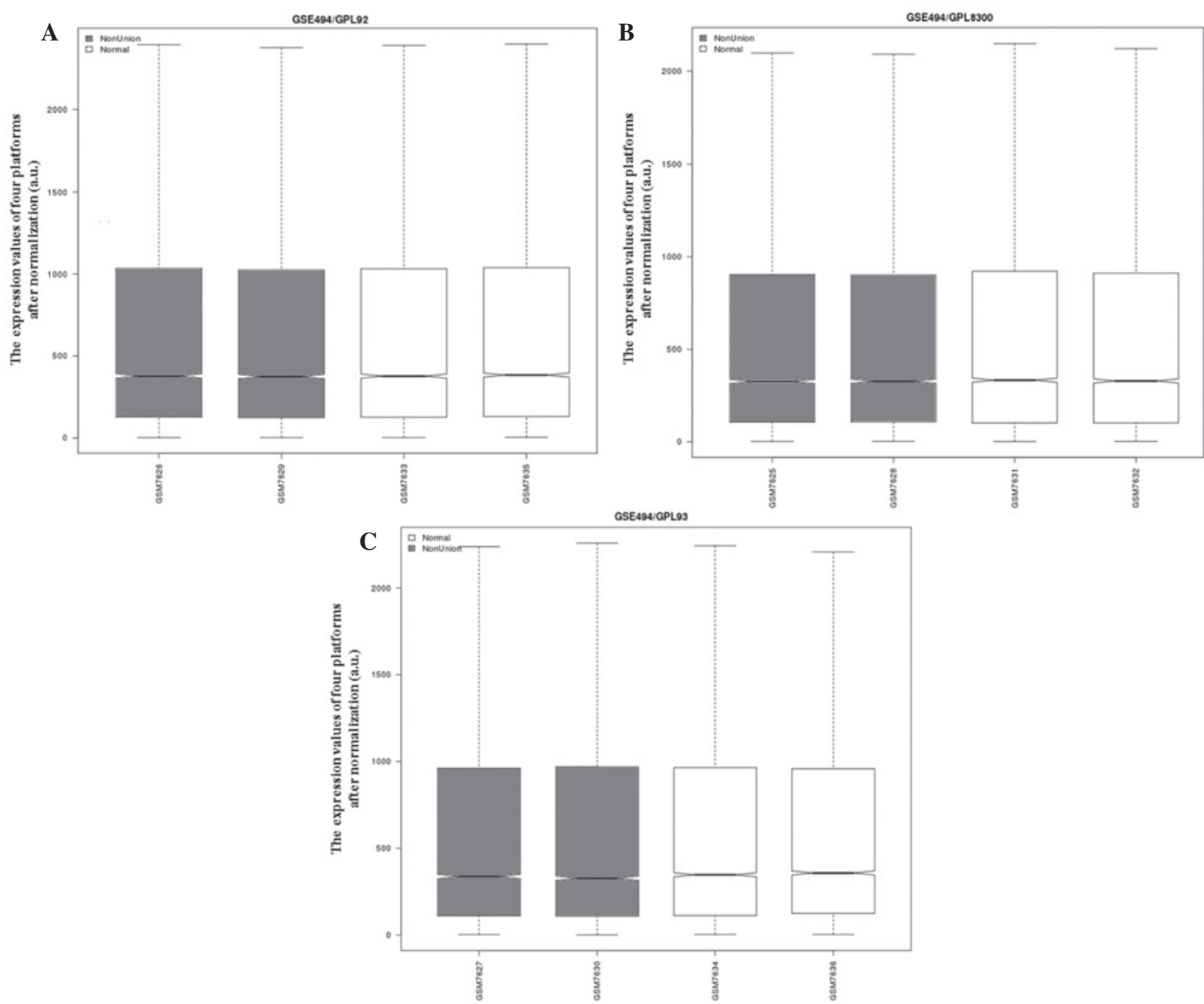

Figure 1. Normalized expression values data box plots. Expression data of the three platforms: (A) GPL92; (B) GPL8300; and (C) GPL93. The gray boxes indicate a non-union fracture sample of the platform, while the white ones indicate normal healing fracture samples. The box in the black line is the median of each set of data, which determines the degree of standardization of data through its distribution. The black lines in the boxes are almost in the same straight line, indicating a good degree of standardization.

Although clinical experience in the treatment of fracture non-unions is quite extensive, studies concerning the high-throughput screening and function identification of differential gene expression associated with fracture non-union are limited. The objective of this study was to document the feature genes and their interacting genes, also further explore their potential functions associated with nonunion fractures.

\section{Materials and methods}

Affymetrix microarray data. The gene chip GSE494 was downloaded from the gene expression database Gene Expression Omnibus (http://www.ncbi.nlm.nih.gov/geo/) and is based on three platforms: GPL92, [HG_U95B] Affymetrix Human Genome U95B Array; GPL93, [HG_U95C] Affymetrix Human Genome U95C Array; and GPL8300, [HG_U95Av2] Affymetrix Human Genome U95 Version 2 Array. There were data for 12 bone samples of fractures in total in the three platforms, with each platform containing data for two normal healing fracture samples and two non-union fracture samples. All the original files and the platform probe annotation information files were also downloaded

Data preprocessing and gene differences analysis. The original data were preprocessed using the R language Affy software package $(8,9)$. The $\mathrm{R}$ language limma package (http://www.r-project.org/) was used to analyze for differentially expressed genes between all the normal and non-union samples (10), and Bayesian methods were used to conduct multiple testing correction. The threshold values were set as $\mathrm{P}<0.05$ and $|\log \mathrm{FC}|>1$.

Predicting the interactions of differentially expressed genes. A single gene is not able to regulate function; only protein-protein interactions (PPIs) have been marked as the main actors for all of the processes taking place in a 
cell and therefore great efforts have been focused towards understanding their biological function (11). Hence, the software HitPredict (http://hintdb.hgc.jp/htp/) was used to analyze the interactions between the differentially expressed genes $(12,13)$. The HitPredict database was built by collecting information from the IntAct BIOGRID and HPRD databases through high-throughput or small-scale experiments of protein interaction associations and, according to the interaction score, estimating protein interactions (the interaction score is obtained according to a likelihood algorithm which uses a Bayesian network combining binding sequence, structure and functional annotations of the PPI in the calculation) (13). HitPredict collects PPI data from high-throughput, small-scale experiments, and considers a likelihood ratio of the resulting score of $>1$ as a high degree of confidence interaction (12). It has collected 239,584 PPIs from nine species, including humans and mice, of which 168,458 are predicted to have a high degree of confidence. The high degree of confidence interactions from the database were used in this study (experimental and likelihood ratio $>1$ ) to analyze the differential gene product.

Enrichment analysis of genes. Differentially expressing genes were screened using enrichment analysis based on the hypergeometric distribution algorithm of FuncAssociate (14). The threshold value of $\mathrm{P}<0.05$ was selected.

Analysis for pathways involving genes in the interaction network. Proteins in a PPI network and the same module usually complete the same biological processes and functions by co-expression. In the present study, enrichment analysis using WebGestalt $(15,16)$, which is based on the hypergeometric distribution algorithm, was used to analyze the pathway of interaction networks involving the differentially expressed gene and its interactions $(\mathrm{P}<0.05)$.

\section{Results}

Screening for differentially expressed genes. The differences between the normalized expression data were compared following data preprocessing (Fig. 1). A total of 531, 418 and 914 genes from the three platforms that met the difference threshold $(\mathrm{P}<0.05$ and $|\log \mathrm{FC}|>1)$ were screened. Two genes, CLU and TSPAN2, were identified to be the commonly differentially expressed genes in the three platforms. The expression values of these two genes were upregulated in the delayed healing fracture sample (Fig. 2).

Predicting the interactions of the differentially expressed genes. Using the software HitPredict to screen for all differentially expressed genes and their product interactions, the gene CLU and 44 interaction objects were obtained (Fig. 3; there were no interaction records of TSPAN2 recorded in HitPredict). The interaction objects of CLU and their likelihood of interaction scores are listed in Table I.

Enrichment analysis of network genes. Enrichment analysis based on the hypergeometric distribution algorithm was conducted using FuncAssociate. A threshold value of $\mathrm{P}<0.05$ was selected. As presented in Table II, five significantly
Table I. Genes interacting with CLU.

\begin{tabular}{|c|c|}
\hline Interactors & Likelihood of interaction \\
\hline TGFR2 & 999 \\
\hline KU70 & 999 \\
\hline TGFR1 & 999 \\
\hline PON1 & 999 \\
\hline PRIO & 999 \\
\hline VLDLR & 999 \\
\hline $\mathrm{C} 7$ & 999 \\
\hline LRP8 & 999 \\
\hline MMP25 & 999 \\
\hline A4 & 999 \\
\hline $\mathrm{C} 8 \mathrm{~B}$ & 999 \\
\hline LEPR & 999 \\
\hline CLUA1 & 999 \\
\hline C9 & 999 \\
\hline LEP & 999 \\
\hline COMD1 & 999 \\
\hline LRP2 & 8.68 \\
\hline DISC1 & 3.37 \\
\hline TNIK & 3.37 \\
\hline APOA1 & 3.37 \\
\hline GRB2 & 3.37 \\
\hline $\mathrm{H} 2 \mathrm{AX}$ & 3.37 \\
\hline NR4A1 & 3.37 \\
\hline FOS & 3.37 \\
\hline MDM2 & 3.37 \\
\hline GCR & 3.37 \\
\hline PPARG & 3.37 \\
\hline ZNF24 & 3.37 \\
\hline B2CL1 & 3.37 \\
\hline MK09 & 3.37 \\
\hline RL23 & 3.37 \\
\hline BAT3 & 3.37 \\
\hline CYP2E1 & 3.37 \\
\hline RBBP8 & 3.37 \\
\hline KLF11 & 3.37 \\
\hline KEAP1 & 3.37 \\
\hline T22D4 & 3.37 \\
\hline SYVN1 & 3.37 \\
\hline UBC & 3.37 \\
\hline NFKB1 & 3.37 \\
\hline IKBA & 3.37 \\
\hline CUL1 & 3.37 \\
\hline FBW1A & 3.37 \\
\hline RAD21 & 3.37 \\
\hline
\end{tabular}

enriched features were obtained. The most significantly enriched genes in the network were associated with sterol transport.

Analyzing gene functions in the co-expression interaction network. By using WebGestalt, which is based on the hyper- 
A

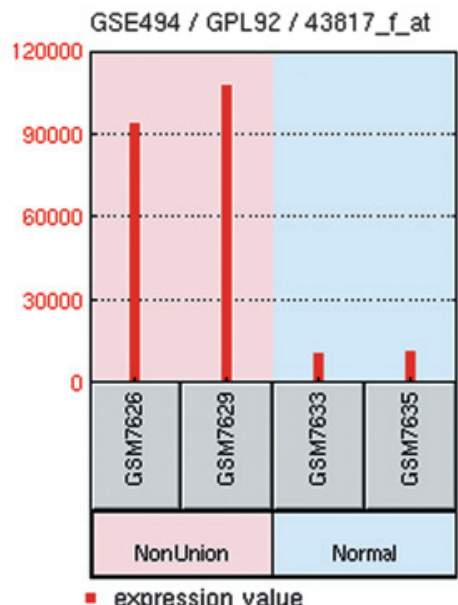

B

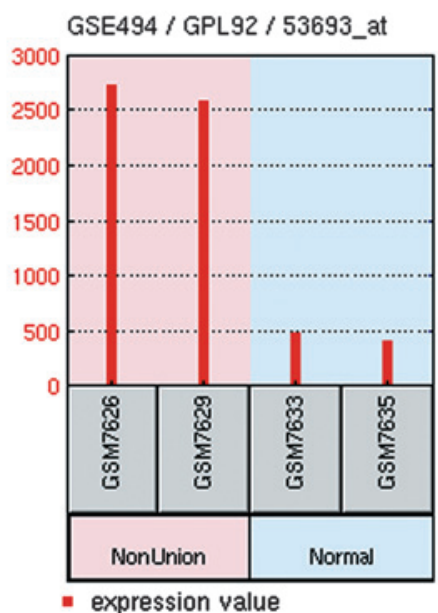

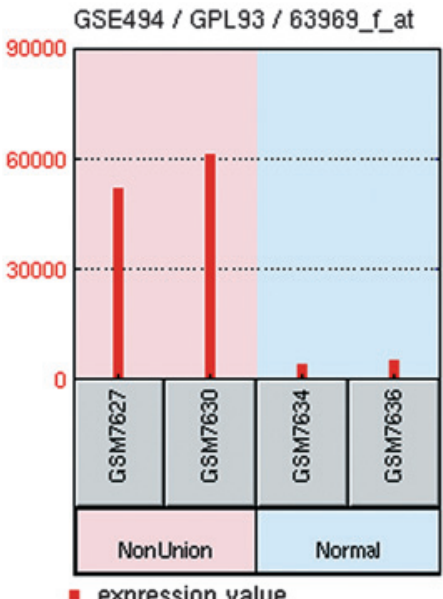

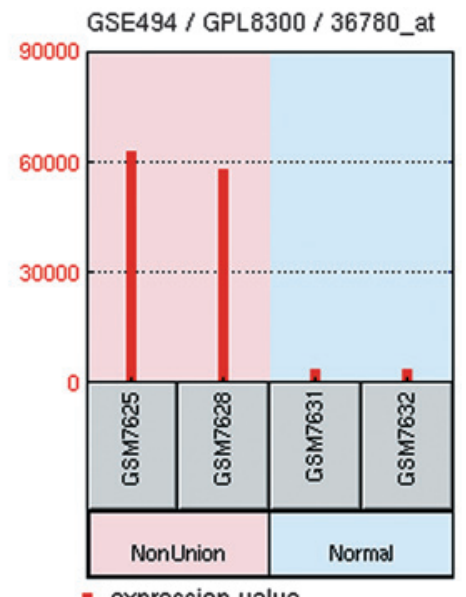

- expression value
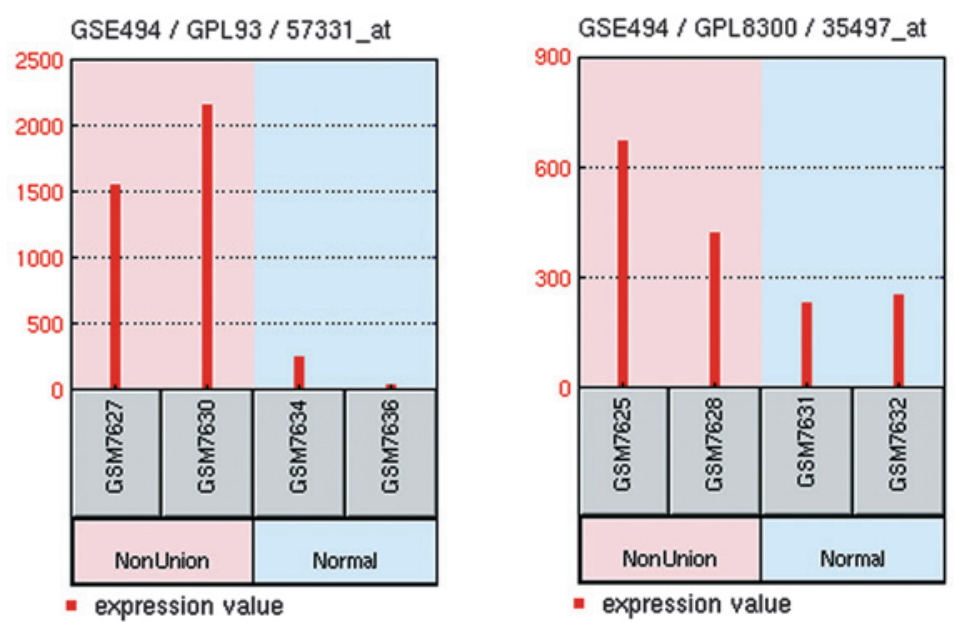

Figure 2. Expression data of the two differentially expressed genes. The expression data of (A) CLU and (B) TSPAN2 in the three platforms. The X-axis indicates grouping and the $\mathrm{y}$-axis demonstrates expression data (a.u.). The height of the red column represents the expression value.

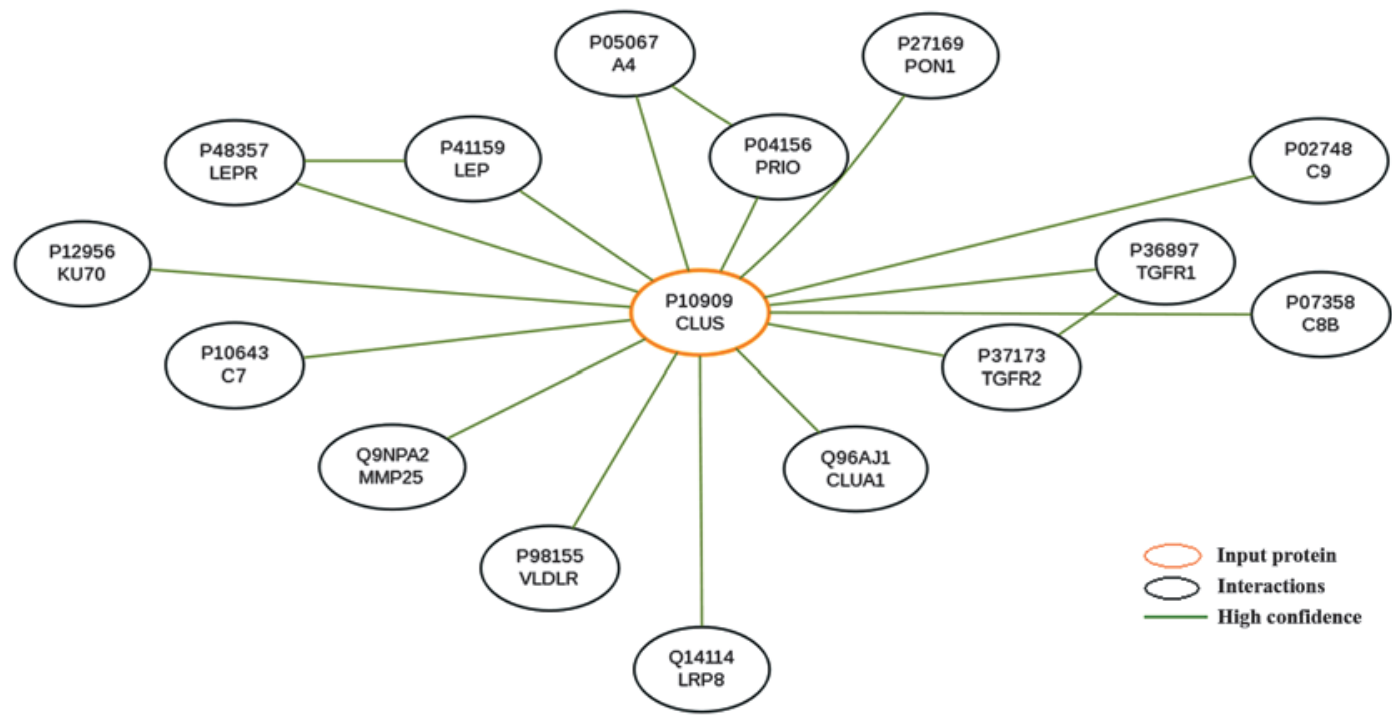

Figure 3. Co-expression network of CLU. The orange circle indicates the input gene CLU, and the black circles indicate interaction objects.

geometric distribution algorithm, to analyze the pathway of the interaction network involving the differentially expressed gene and its interactors (threshold value $\mathrm{P}<0.05$ ), four pathways with significantly enriched genes were identified (Table III). One of the most significant pathways was osteoclast differentiation, which involved the SYVN1, MDM2, KEAP1 and CLU genes. 
Table II. List of functions associated with enriched genes in the network.

\begin{tabular}{|c|c|c|c|}
\hline ID & Term & P-value & Genes \\
\hline GO:0032371 & Regulation of sterol transport & 0.00000703 & LEP, APOA1, PON1, NFKB1,CLU \\
\hline GO:0044421 & Extracellular region part & 0.0000341 & $\begin{array}{l}\text { LEP, C8B, APOA1, LEPR, CLU, PON1, LRP8, LRP2, } \\
\text { MMP25, VLDLR }\end{array}$ \\
\hline GO:0005576 & Extracellular region & 0.000481 & $\begin{array}{l}\text { LEP, C8B, C7, APOA1, C9, LEPR, CLU, PON1, LRP8, } \\
\text { LRP2, MMP25, VLDLR }\end{array}$ \\
\hline GO:0043233 & Organelle lumen & 0.004086798 & $\begin{array}{l}\text { FOS, APOA1, SYVN1, CLU, UBC, NR4A1, MDM2, } \\
\text { NFKB1, KEAP1, CUL1 }\end{array}$ \\
\hline GO:0031974 & Membrane-enclosed lumen & 0.004673635 & $\begin{array}{l}\text { FOS, APOA1, SYVN1, CLU, UBC, NR4A1, MDM2, } \\
\text { NFKB1, KEAP1, CUL1 }\end{array}$ \\
\hline
\end{tabular}

Table III. List of pathways associated with enriched genes in the network.

\begin{tabular}{llll}
\hline ID & \multicolumn{1}{c}{ Term } & \multicolumn{1}{c}{ P-value } & \multicolumn{1}{c}{ Genes } \\
\hline hsa04380 & Osteoclast differentiation & 0.011605668 & SYVN1, MDM2, KEAP1, CUL \\
hsa04920 & Adipocytokine signaling pathway & 0.022842413 & LEP, LEPR, NFKB1 \\
hsa04610 & Complement and coagulation cascades & 0.024136338 & C8B, C7, C9 \\
hsa04662 & B cell receptor signaling pathway & 0.028195492 & FOS, GRB2, NFKB1
\end{tabular}

\section{Discussion}

Non-union of a fracture is defined as the cessation of all reparative processes of healing without bone-union (17). As previous data has demonstrated that the diagnosis of non-union fractures is based on clinical symptoms and physical findings, including pain at the fracture site and evidence of pathologic motion (18), there are seldom studies on the high-throughput screening and function identification of differential gene expression in fracture non-unions. In the present study, the upregulated gene CLU and its 44 interaction objects were selected from a microarray chip composed of normal union and non-union skeletal fracture samples. According to the function of CLU and its interacting genes, the conclusion was reached that by inhibiting the normal healing process following a fracture, the selected genes regulated the healing of non-union skeletal fractures through participating in sterol transport and the pathway involved in the differentiation of osteoclasts.

CLU (also known as clusterin, apolipoprotein J, TRPM-2 and SGP-2) is highly conserved in different species, with approximately $70-80 \%$ protein homology in mammals with other species. CLU consists of a 449-amino-acid primary polypeptide chain. By its disulfide bridges, human CLU is cleaved into $\alpha$ and $\beta$ chains (19). CLU expression is low in normal conditions but is induced by stress stimuli, suggesting that its function may be directly or indirectly associated with the stress response (20). In a number of studies, CLU has been demonstrated to be antiapoptotic, protecting cells against a variety of death signals (21-23). Although there have been no direct studies indicating that CLU is associated with fracture healing, a recent study has demonstrated that the mRNA levels of CLU are increased in early osteoarthritic articular
(OA) cartilage, while they are decreased in advanced OA (24). IL- $1 \alpha$-stimulated cartilage explants have been demonstrated to produce decreased levels of CLU compared with those in untreated cartilage (25) and treatment with IL-1 $\beta$ also decreases the levels of CLU (26). Synovial apoptosis inhibitor 1 (SYVN1), also known as DER3 and HRD1, is an E3 ubiquitin ligase that is implicated in endoplasmic reticulum-associated degradation (27). It is cloned from rheumatoid synovial cells and is highly expressed in the synoviocytes of patients with rheumatoid arthritis (RA). Through its antiapoptotic effect, SYVN1 promotes the overproliferation of synoviocytes $(28,29)$. Murine double minute 2 ( $\mathrm{mdm} 2$ ) was first identified as the gene responsible for the spontaneous transformation of $3 \mathrm{~T} 3$ cells (30). As an E3 ubiquitin ligase, $\mathrm{mdm} 2$ is a critical negative regulator of p53 by targeting it for ubiquitination and proteasomal degradation (31). Individuals carrying the mdm2 SNP309 T/G or $\mathrm{G} / \mathrm{G}$ have been identified to exhibit a significantly earlier age of onset for osteosarcoma (32). In RA patients, the frequencies of the mdm2 SNP309 are significantly reduced (33), while the mdm2 SNP 309G/G is associated with higher levels of apoptotic activity in RA-derived synoviocytes (34). Kelch-like ECH associated protein 1 (Keap1) is a stress sensor and an adaptor component of Cullin 3-based E3 ubiquitin ligase (35). Under normal (unstressed) conditions, Keap1 activates and rapidly degrades Nrf2 through the proteasome pathway. Upon cellular exposure, as an E3 ubiquitin ligase component, Keap1 is inhibited, which provokes Nrf2 stabilization (36). A study has reported that the Nrf2-Keap1 signaling cascade is conserved in human skeletal muscle (37).

Fracture healing is a complex process that involves osteoblasts, osteoclasts and a variety of other cells and cytokines (38), which means it may be the outcome of the interaction of multiple genes. Although there have been only indirect 
studies that have indicating that CLU and its interacting genes are involved in healing fractures, there is significant evidence that they participate in the healing process. In conclusion, the generally stronger inhibition of osteoblasts in non-union fractures (39) combined with the results of the present study indicating that CLU and its interacting genes SYVN1, MDM2 and KEAP1 participate in the osteoclast differentiation pathway suggest that all the genes which were identified by screening may regulate the healing of fractures through an involvement in osteoclast differentiation.

\section{Acknowledgements}

This study was supported by the National Natural Science Foundation of China (grant no. C05030324).

\section{Reference}

1. Arthritis and related conditions. In: United States Bone and Joint Decade: The Burden of Musculoskeletal Diseases in the United States. 1st edition. American Academy of Orthopaedic Surgeons, Rosemont, IL, pp99-161, 2008.

2. Einhorn TA: The cell and molecular biology of fracture healing. Clin Orthop Relat Res 355 (Suppl): S7-S21, 1998.

3. Ai-Aql ZS, Alagl AS, Graves DT, Gerstenfeld LC and Einhorn TA: Molecular mechanisms controlling bone formation during fracture healing and distraction osteogenesis. J Dent Res 87: 107-118, 2008.

4. Gerstenfeld LC, Cullinane DM, Barnes GL, Graves DT and Einhorn TA: Fracture healing as a post-natal developmental process: molecular, spatial, and temporal aspects of its regulation. J Cell Biochem 88: 873-884, 2003.

5. Ferguson C, Alpern E, Miclau T and Helms JA: Does adult fracture repair recapitulate embryonic skeletal formation? Mech Dev 87: 57-66, 1999.

6. Hofmann A, Ritz U, Hessmann MH, et al: Cell viability, osteoblast differentiation, and gene expression are altered in human osteoblasts from hypertrophic fracture non-unions. Bone 42: 894-906, 2008.

7. Megas P: Classification of non-union. Injury 36 (Suppl 4): S30-S37, 2005.

8. Troyanskaya O, Cantor M, Sherlock G, et al: Missing value estimation methods for DNA microarrays. Bioinformatics 17: 520-525, 2001.

9. Fujita A, Sato JR, Rodrigues Lde O, Ferreira CE and Sogayar MC: Evaluating different methods of microarray data normalization. BMC Bioinformatics 7: 469, 2006.

10. Smyth GK: Limma: Linear models for microarray data. In: Bioinformatics and Computational Biology Solution Using R and Bioconductor. Statistics for Biology and Health. Gentleman R, Carey VJ, Huber W, Irizarry RA and Dudoit S (eds). Springer, New York, NY, pp397-420, 2005.

11. Tong AH, Drees B, Nardelli G, et al: A combined experimental and computational strategy to define protein interaction networks for peptide recognition modules. Science 295: 321-324, 2002.

12. Patil A and Nakamura H: Filtering high-throughput protein-protein interaction data using a combination of genomic features. BMC Bioinformatics 6: 100, 2005.

13. Patil A, Nakai K and Nakamura H: HitPredict: a database of quality assessed protein-protein interactions in nine species. Nucleic Acids Res 39: D744-D749, 2011.

14. Berriz GF, Beaver JE, Cenik C, Tasan M and Roth FP: Next generation software for functional trend analysis. Bioinformatics 25: 3043-3044, 2009.

15. Zhang B, Kirov S and Snoddy J: WebGestalt: an integrated system for exploring gene sets in various biological contexts. Nucleic Acids Res 33: W741-W748, 2005.

16. Duncan D, Prodduturi N and Zhang B: WebGestalt2: an updated and expanded version of the Web-based Gene Set Analysis Toolkit. BMC Bioinformatics 11 (Suppl 4): P10, 2010.

17. Cleveland KB: Delayed union and nonunion of fractures. In: Campbell's Operative Orthopaedics. Crenshaw AH (ed). Vol 3. 7th edition. CV Mosby, St. Louis, MO, pp2053-2118, 1987.
18. McKee MD: Aseptic non-union. In: AO Principles of Fracture Management. Rüedi TP and Murphy WM (eds). 1st edition. Georg Thieme Verlag, Stuttgart and New York, pp748-762, 2000.

19. Jones SE and Jomary C: Clusterin. Int J Biochem Cell Biol 34: 427-431, 2002.

20. McLaughlin L, Zhu G, Mistry M, et al: Apolipoprotein J/clusterin limits the severity of murine autoimmune myocarditis. J Clin Invest 106: 1105-1113, 2000.

21. Chaiwatanasirikul K and Sala A: The tumour-suppressive function of CLU is explained by its localisation and interaction with HSP60. Cell Death Dis: 2, e219, 2011.

22. Trougakso IP, So A, Jansen B, Gleave ME and Gonos ES: Silencing expression of the clusterin/apolipoprotein J gene in human cancer cells using small interfering RNA induces spontaneous apoptosis, reduced growth ability, and cell sensitization to genotoxic and oxidative stress. Cancer Res 64: 1834-1842, 2004.

23. Zellweger T, Chi K, Miyake H, Adomat H, Kiyama S, Skov K and Gleave ME: Enhanced radiation sensitivity in prostate cancer by inhibition of the cell survival protein clusterin. Clin Cancer Res 8: 3276-3284, 2002.

24. Connor JR, Kumar S, Sathe G, et al: Clusterin expression in adult human normal and osteoarthritic articular cartilage. Osteoarthritis Cartilage 9: 727-737, 2001.

25. Wilson R, Belluoccio D, Little CB, Fosang AJ and Bateman JF: Proteomic characterization of mouse cartilage degradation in vitro. Arthritis Rheum 58: 3120-3131, 2008.

26. Clutterbuck AL, Smith JR, Allaway D, Harris P, Liddell S and Mobasheri A: High throughput proteomic analysis of the secretome in an explant model of articular cartilage inflammation. J Proteomics 74: 704-715, 2011.

27. Yamasaki S, Yagishita N, Sasaki T, et al: Cytoplasmic destruction of p53 by the endoplasmic reticulum-resident ubiquitin ligase 'Synoviolin'. EMBO J 26: 113-122, 2007.

28. Amano T, Yamasaki S, Yagishita N, et al: Synoviolin/Hrd1, an E3 ubiquitin ligase, as a novel pathogenic factor for arthropathy. Genes Dev 17: 2436-2449, 2003.

29. Tsuchimochi K, Yagishita N, Yamasaki S, et al: Identification of a crucial site for synoviolin expression. Mol Cell Biol 25: 7344-7356, 2005.

30. Cahilly-Snyder L, Yang-Feng T, Francke U and George DL: Molecular analysis and chromosomal mapping of amplified genes isolated from a transformed mouse 3T3 cell line. Somat Cell Mol Genet 13: 235-244, 1987.

31. Harris SL and Levine AJ: The p53 pathway: positive and negative feedback loops. Oncogene 24: 2899-2908, 2005.

32. Bond GL, Hu W, Bond EE, et al: A single nucleotide polymorphism in the MDM2 promoter attenuates the p53 tumor suppressor pathway and accelerates tumor formation in humans. Cell 119: 591-602, 2004.

33. Assmann G, Voswinkel J, Mueller M, et al: Association of rheumatoid arthritis with Mdm2 SNP309 and genetic evidence for an allele-specific interaction between MDM2 and p53 P72R variants: a case control study. Clin Exp Rheumatol 27: 615-619, 2009.

34. Heyne K, Huwer J, Zimmer V, Pfreundschuh M, Ney JT and Assmann G: Different apoptotic responses of RA synoviocytes depending on different genotypes of the mdm2 SNP T309G. Apoptosis 17: 424-427, 2012.

35. Kobayashi A, Kang MI, Okawa H, et al: Oxidative stress sensor Keap1 functions as an adaptor for Cul3-based E3 ligase to regulate proteasomal degradation of Nrf2. Mol Cell Biol 24: 7130-7139, 2004

36. Seibenhener ML, Geetha $\mathrm{T}$ and Wooten MW: Sequestosome 1/p62 - more than just a scaffold. FEBS Lett 581: 175-179, 2007.

37. Safdar A, deBeer J and Tarnopolsky MA: Dysfunctional Nrf2-Keap1 redox signaling in skeletal muscle of the sedentary old. Free Radic Biol Med 49: 1487-1493, 2010.

38. Lories RJ, Daans M, Derese I, et al: Noggin haploinsufficiency differentially affects tissue responses in destructive and remodeling arthritis. Arthritis Rheum 54: 1736-1746, 2006.

39. Kloen P, Lauzier D and Hamdy RC: Co-expression of BMPs and BMP-inhibitors in human fractures and non-unions. Bone 51: 59-68, 2012. 